Check for updates

Cite this: Phys. Chem. Chem. Phys., 2019, 21, 7491

Received 20th February 2019, Accepted 13th March 2019

DOI: $10.1039 / c 9 c p 01014 a$

rsc.li/pccp

\section{A unified theory of weak-field coherent control of the behavior of a resonance state}

\begin{abstract}
A. García-Vela (D)
A unified weak-field control scheme to modify the two properties that determine the whole behavior of a resonance state, namely the lifetime and the asymptotic fragment distribution produced upon resonance decay, is proposed. Control is exerted through quantum interference induced between overlapping resonances of the system, by exciting two different energies at which the resonances overlap. The scheme applies a laser field consisting of a first pulse that excites the energy of the resonance to be controlled, and two additional pulses that excite another different energy to induce interference, with a delay time with respect to the first pulse. Each of the two additional pulses is used to control one of the two resonance properties, by adjusting its corresponding delay time: with a relatively short delay time the second pulse controls the resonance lifetime, while with a very long delay time the third pulse modifies the asymptotic fragment distribution produced. The efficiency of the control of each resonance property is found to be strongly dependent on the choice of the second interfering energy, which allows for a more flexible control optimization by choosing a different energy for each property. The theory underlying the interference mechanism of the control scheme is developed and presented, and is applied to analyze and explain the results obtained. The present scheme thus appears to be a useful tool for controlling resonance-mediated molecular processes.
\end{abstract}

\section{Introduction}

Resonance states govern a large variety of molecular processes that occur upon resonance decay. ${ }^{1,2}$ Among them are photodissociation processes like electronic, ${ }^{3}$ vibrational, ${ }^{4,5}$ and rotational $^{6}$ predissociation of a molecular system, as well as lowtemperature reactive ${ }^{7-13}$ and nonreactive ${ }^{14-21}$ molecular collisions. Control of such molecular processes has been actively pursued in the last few decades. ${ }^{22-34}$ In the case of resonance-mediated processes, their control is closely related to the control of the underlying resonance decay process. And the behavior of this decay process is determined essentially by two resonance properties which are the lifetime (which determines the duration of the process) and the product fragment state distribution produced upon the decay (which determines the outcome of the process). Thus, modifying these resonance properties provides an effective means of control over the resonance-mediated process of interest.

Strategies to control the resonance lifetime based on quantum interference have been suggested in environments of both overlapping and isolated resonance states in the weak-field regime. ${ }^{35-39}$ Quantum interference was also the basis of different weak-field control schemes proposed to modify the fragment distribution

Instituto de Física Fundamental, Consejo Superior de Investigaciones Cientificas, Serrano 123, 28006 Madrid, Spain. E-mail: garciavela@iff.csic.es produced upon resonance decay. ${ }^{40-44}$ Some of the above control schemes share the common feature that they apply a pump laser field consisting of two pulses with a time delay between them. ${ }^{36,37,39,42-44}$ Each of these two pulses is used to excite a different resonance energy in an environment of overlapping resonances, which then interfere. By varying the time delay between the pulses, the resonance interference can be controlled. Depending on how long is the time delay between the pulses, control of a different resonance property (either the lifetime or the fragment distribution) is achieved. More specifically, if the time delay between the pulses is relatively short, such that the two resonances are simultaneously populated in time, the lifetime of both resonances can be controlled. ${ }^{36,37,39}$ However, when the time delay is long enough such that the population of the resonance excited with the first pulse has decayed completely when the other resonance is excited with the second pulse, then the product fragment distribution associated with the first resonance can be modified in the asymptotic time regime. ${ }^{44}$ Thus, the time delay applied between pulses is related to the nature and time scale of the resonance property under control.

So far the above type of control scheme has been applied to modify separately either the resonance lifetime or the fragment distribution. However, it is possible - and desirable - to design a unified control scheme that allows for the control of the two resonance properties determining the behavior of a resonancemediated molecular process. Indeed this can be done by applying 
a laser field consisting of several pulses, where the first pulse would excite the resonance to be controlled, while the subsequent pulses would be delayed with respect to the first one accordingly to the dynamical time scale of the resonance property which is to be modified. The practical advantage of such a unified scheme is that the behavior along the whole course of the process of interest can be controlled within a single experiment, just by choosing appropriately the time delay of the pulses that control each resonance property. The main goal of the present work is to demonstrate the possibility of this unified control scheme both numerically and formally, by developing the underlying equations of the theory. As in earlier works the scheme is applied to the vibrational predissociation of the $\mathrm{Ne}-\mathrm{Br}_{2}\left(\mathrm{~B}, \nu^{\prime}\right)$ complex, because this system features overlapping resonances in different regimes. Resonances are common to several types of weakly bound complexes. ${ }^{45}$

This paper is organized as follows. Section 2 presents the main features of the methodology applied, and the formal theory underlying the control scheme. In Section 3 the results are presented and discussed. Conclusions are given in Section 4 .

\section{Theory}

Upon laser excitation, $\mathrm{Ne}-\mathrm{Br}_{2}\left(\mathrm{X}, \nu^{\prime \prime}=0\right)+h \nu \rightarrow \mathrm{Ne}-\mathrm{Br}_{2}\left(\mathrm{~B}, \nu^{\prime}, n^{\prime}\right)$, an intermolecular van der Waals resonance $n^{\prime}$ of $\mathrm{Ne}-\mathrm{Br}_{2}\left(\mathrm{~B}, \nu^{\prime}\right)$ is populated. The labels $\nu^{\prime \prime}$ and $\nu^{\prime}$ denote the vibrational states of $\mathrm{Br}_{2}$ in the $\mathrm{X}$ and $\mathrm{B}$ electronic states, respectively, while the $n^{\prime}$ index labels the energy position of the resonance, with $n^{\prime}=0$ corresponding to the ground one. Then the resonance excited decays to the fragmentation continuum through vibrational predissociation, $\mathrm{Ne}-\mathrm{Br}_{2}\left(\mathrm{~B}, \nu^{\prime}, n^{\prime}\right) \rightarrow \mathrm{Ne}+\mathrm{Br}_{2}\left(\mathrm{~B}, \nu_{\mathrm{f}}<\nu^{\prime}\right)$. This process has been studied in detail both experimentally ${ }^{46,47}$ and theoretically. ${ }^{4,5}$

The $\mathrm{Ne}-\mathrm{Br}_{2}\left(\mathrm{~B}, \nu^{\prime}, n^{\prime}\right)$ excitation with a laser field and the subsequent predissociation were simulated with a full threedimensional wave packet method (assuming $J=0$ ) described in detail elsewhere. ${ }^{4,35}$ In order to assess the quality of the model applied, it is noted that the lifetime calculated with the present theoretical model for the decay of the $\mathrm{Ne}-\mathrm{Br}_{2}\left(\mathrm{~B}, \nu^{\prime}=16\right)$ ground intermolecular resonance was found to be $69 \mathrm{ps},{ }^{38}$ while the corresponding lifetime estimated experimentally is $68 \pm 3 \mathrm{ps}^{47}$ This good agreement implies that both the three-dimensional wave packet method and the potential surfaces used in the present simulations are realistic enough in order to describe this resonance decay process.

In what follows the formal theory underlying the unified control scheme proposed in this work is developed. Let $\hat{H}$ be the total Hamiltonian of a molecular system that supports resonances. Following the discussion on the decay of a resonance state of Cohen-Tannoudji et al., ${ }^{48}$ we can write $\hat{H}$ as $\hat{H}=\hat{H}_{0}+W$, where $\hat{H}_{0}$ is a zeroth-order Hamiltonian and $W$ is a coupling. The spectrum of $\hat{H}_{0}$ consists of a set of discrete bound states $\chi_{i}$ (located in the interaction region) with associated energies $E_{i}$, and a set of continuum states $\varphi_{E, m}$ (associated with the product fragments in the asymptotic region) with associated energies $E$ and with $m$ a global label for the fragment internal states. When
$W=0$ the $\chi_{i}$ states are true bound states, but when $W \neq 0, \chi_{i}$ become resonances $\psi_{i}$ that decay to the continuum of $\varphi_{E, m}$ states. These states fulfill the orthogonality relations

$$
\left\langle\chi_{i} \mid \chi_{j}\right\rangle=\delta_{i j}, \quad\left\langle\varphi_{E^{\prime}, m^{\prime}} \mid \varphi_{E, m}\right\rangle=\delta_{m^{\prime} m} \delta\left(E^{\prime}-E\right), \quad\left\langle\chi_{i} \mid \varphi_{E, m}\right\rangle=0
$$

and form a complete basis set, in terms of which the state of the system excited at the energy $E$ can be expressed. Let $\psi_{E}$ be the stationary eigenstates of Hamiltonian $\hat{H}$ associated with energy $E$ in the excited electronic state. Such eigenstates (which also form a complete basis set) can be expanded in the set of the $\chi_{i}$ and $\varphi_{E, m}$ states as

$$
\psi_{E}(t)=\sum_{k} A_{k}^{(E)} \chi_{k} \mathrm{e}^{-i E_{k} t / \hbar}+\sum_{m^{\prime}} \int \mathrm{d} E^{\prime} B_{E^{\prime}, m^{\prime}}^{(E)} \varphi_{E^{\prime}, m^{\prime}} \mathrm{e}^{-i E^{\prime} t / \hbar},
$$

where the above summation over $k$ considers the possibility that in general more than one state $\chi_{k}$ may contribute to $\psi_{E}(t)$ in an environment of overlapping resonances. The time dependence of $\psi_{E}(t)$ is the trivial one (i.e., $\left|\psi_{E}(t)\right|^{2}$ is time independent, as can be easily checked by applying the relations of eqn (1)).

Let us now assume that within a vibrational manifold $\nu^{\prime}$ of $\mathrm{Ne}-\mathrm{Br}_{2}\left(\mathrm{~B}, \nu^{\prime}\right)$ featuring a group of intermolecular overlapping resonances, a given resonance energy $E_{\mathrm{a}}$ is excited with an electric field consisting of a Gaussian-shaped pulse, $\mathcal{E}_{1}(t)=A_{1} \mathrm{e}^{-\left(t-t_{1}\right)^{2} / 2 \sigma^{2}}$ $\cos \left[\omega_{1}\left(t-t_{1}\right)+\phi_{1}\right]$, where $A_{1}$ is the pulse amplitude, $t_{1}$ is the time center of the pulse, $\sigma$ is related to the pulse temporal width, $\omega_{1}$ is the photon frequency required to excite the resonance energy $E_{\mathrm{a}}$ from the ground vibronic state $\mathrm{Ne}-\mathrm{Br}_{2}\left(\mathrm{X}, \nu^{\prime \prime}=0, n^{\prime \prime}=0\right)$, and $\phi_{1}$ is the pulse phase. It is noted that in a framework of overlapping resonances, several resonances may contribute at a given energy $E_{\mathrm{a}}$, one of them providing the dominant contribution. ${ }^{49}$ Excitation of the system by $\mathcal{E}_{1}(t)$ creates a wave packet $\xi_{E_{\mathrm{a}}}(t)$ that can be expressed as

$$
\begin{aligned}
\xi_{E_{\mathrm{a}}}(t)= & \int \mathrm{d} E^{\prime \prime} C_{E^{\prime \prime}}^{\left(E_{\mathrm{a}}\right)}(t) \psi_{E^{\prime \prime}}(t)=\sum_{k} a_{k}^{\left(E_{\mathrm{a}}\right)}(t) \chi_{k} \mathrm{e}^{-i E_{k} t / \hbar} \\
& +\sum_{m^{\prime}} \int \mathrm{d} E^{\prime} b_{E^{\prime}, m^{\prime}}^{\left(E_{\mathrm{a}}\right)}(t) \varphi_{E^{\prime}, m^{\prime}} \mathrm{e}^{-i E^{\prime} t / \hbar},
\end{aligned}
$$

where $\quad a_{k}^{\left(E_{\mathrm{a}}\right)}(t)=\int \mathrm{d} E^{\prime \prime} C_{E^{\prime \prime}}^{\left(E_{\mathrm{a}}\right)}(t) A_{k}^{\left(E^{\prime \prime}\right)} \quad$ and $\quad b_{E^{\prime}, m^{\prime}}^{\left(E_{\mathrm{a}}\right)}(t)=$ $\int \mathrm{d} E^{\prime \prime} C_{E^{\prime \prime}}^{\left(E_{\mathrm{a}}\right)}(t) B_{E^{\prime}, m^{\prime}}^{\left(E^{\prime \prime}\right)}$. The basis of the control scheme suggested is to induce quantum interference between the overlapping resonances, and to achieve this goal (at least) two resonance energies are to be excited by applying a laser field with two Gaussian pulses,

$$
\begin{aligned}
\mathcal{E}_{2}(t)= & A_{1} \mathrm{e}^{-\left(t-t_{1}\right)^{2} / 2 \sigma^{2}} \cos \left[\omega_{1}\left(t-t_{1}\right)+\phi_{1}\right] \\
& +A_{2} \mathrm{e}^{-\left(t-t_{2}\right)^{2} / 2 \sigma^{2}} \cos \left[\omega_{2}\left(t-t_{2}\right)+\phi_{2}\right],
\end{aligned}
$$

where $\omega_{1}$ and $\omega_{2}$ are the pulse frequencies corresponding to the excitation of the two resonance energies, and $\Delta t=t_{2}-t_{1}$ is the delay time between the two pulses. When the laser field $\mathcal{E}_{2}(t)$ is applied to excite the resonance energies $E_{\mathrm{a}}$ (excited by the pulse centered at $t_{1}$ ) and $E_{\mathrm{b}}$ (excited by the pulse centered at $t_{2}$ ), a wave packet is created,

$$
\Phi(t)=\xi_{E_{\mathrm{a}}}(t)+\xi_{E_{\mathrm{b}}}(t),
$$


with $\xi_{E_{\mathrm{a}}}(t)$ and $\xi_{E_{\mathrm{b}}}(t)$ being the amplitudes excited around $E_{\mathrm{a}}$ and $E_{\mathrm{b}}$ by the two pulses of $\mathcal{E}_{2}(t)$. We shall assume that the two pulses of $\mathcal{E}_{2}(t)$ are spectrally narrow enough such that they, and therefore $\xi_{E_{\mathrm{a}}}(t)$ and $\xi_{E_{\mathrm{b}}}(t)$, do not overlap in energy. Now, using eqn (3) we can write,

$$
\begin{aligned}
\Phi(t)= & \int \mathrm{d} E^{\prime \prime}\left[C_{E^{\prime \prime}}^{\left(E_{\mathrm{a}}\right)}(t)+C_{E^{\prime \prime}}^{\left(E_{\mathrm{b}}\right)}(t)\right] \psi_{E^{\prime \prime}}(t) \\
= & \sum_{k}\left[a_{k}^{\left(E_{\mathrm{a}}\right)}(t)+a_{k}^{\left(E_{\mathrm{b}}\right)}(t)\right] \chi_{k} \mathrm{e}^{-i E_{k} t / \hbar} \\
& +\sum_{m^{\prime}} \int \mathrm{d} E^{\prime}\left[b_{E^{\prime}, m^{\prime}}^{\left(E_{\mathrm{a}}\right)}(t)+b_{E^{\prime}, m^{\prime}}^{\left(E_{\mathrm{b}}\right)}(t)\right] \varphi_{E^{\prime}, m^{\prime}} \mathrm{e}^{-i E^{\prime} t / \hbar} .
\end{aligned}
$$

\subsection{Control of the resonance lifetime}

As discussed in earlier works, ${ }^{35-39}$ control of the resonance lifetime is achieved by modifying the shape of the resonance survival probability by means of quantum interference. A resonance wave function $\psi_{i}$ can be expressed as

$$
\psi_{i}(t)=\int \mathrm{d} E^{\prime \prime} c_{E^{\prime \prime}}^{(i)} \psi_{E^{\prime \prime}}(t) .
$$

If the target resonance for which we want to control the lifetime is that associated with energy $E_{\mathrm{a}}$, by using eqn (6) and (7) we can express its survival probability $I_{\mathrm{a}}(t)$ as

$$
\begin{aligned}
& I_{\mathrm{a}}(t)=\left|\left\langle\psi_{\mathrm{a}}(t) \mid \Phi(t)\right\rangle\right|^{2} \\
& =\left|\int \mathrm{d} E^{\prime \prime} \int \mathrm{d} E^{\prime} c_{E^{\prime \prime}}^{(\mathrm{a}) *}\left[C_{E^{\prime}}^{\left(E_{\mathrm{a}}\right)}(t)+C_{E^{\prime}}^{\left(E_{\mathrm{b}}\right)}(t)\right]\left\langle\psi_{E^{\prime \prime}}(t) \mid \psi_{E^{\prime}}(t)\right\rangle\right|^{2} \\
& =\left|\int \mathrm{d} E^{\prime} c_{E^{\prime}}^{(\mathrm{a}) *}\left[C_{E^{\prime}}^{\left(E_{\mathrm{a}}\right)}(t)+C_{E^{\prime}}^{\left(E_{\mathrm{b}}\right)}(t)\right]\right|^{2} \\
& =\left|d_{\mathrm{a}}^{\left(E_{\mathrm{a}}\right)}(t)+d_{\mathrm{a}}^{\left(E_{\mathrm{b}}\right)}(t)\right|^{2} \\
& =\left|d_{\mathrm{a}}^{\left(E_{\mathrm{a}}\right)}(t)\right|^{2}+d_{\mathrm{a}}^{\left(E_{\mathrm{a}}\right)}(t) d_{\mathrm{a}}^{\left(E_{\mathrm{b}}\right) *}(t) \\
& +d_{\mathrm{a}}^{\left(E_{\mathrm{a}}\right) *}(t) d_{\mathrm{a}}^{\left(E_{\mathrm{b}}\right)}(t)+\left|d_{\mathrm{a}}^{\left(E_{\mathrm{b}}\right)}(t)\right|^{2},
\end{aligned}
$$

where $d_{\mathrm{a}}^{\left(E_{\mathrm{a}}\right)}(t)=\int \mathrm{d} E^{\prime} c_{E^{\prime}}^{(\mathrm{a}) *} C_{E^{\prime}}^{\left(E_{\mathrm{a}}\right)}(t)$ and $d_{\mathrm{a}}^{\left(E_{\mathrm{b}}\right)}(t)=\int \mathrm{d} E^{\prime} c_{E^{\prime}}^{(\mathrm{a}) *} C_{E^{\prime}}^{\left(E_{\mathrm{b}}\right)}(t)$.

Eqn (8) shows that $I_{\mathrm{a}}(t)$ consists of four terms. The first term, $\left|d_{\mathrm{a}}^{\left(E_{\mathrm{a}}\right)}(t)\right|^{2}$, is the survival probability that would be obtained if a single resonance energy $E_{\text {a }}$ was excited with the $\mathcal{E}_{1}(t)$ field. The lifetime associated with this $I_{\mathrm{a}}(t)=\left|d_{\mathrm{a}}^{\left(E_{\mathrm{a}}\right)}(t)\right|^{2}$ survival probability would be the "isolated resonance lifetime", $\tau_{\mathrm{a}}^{\text {iso }}$. The three additional terms of eqn (8) arise from the excitation of amplitude at the second energy $E_{\mathrm{b}}$ and its interference with the resonance amplitude excited at $E_{\mathrm{a}}$. Those three terms are nonzero as long as the $d_{\mathrm{a}}^{\left(E_{\mathrm{a}}\right)}(t)$ and $d_{\mathrm{a}}^{\left(E_{\mathrm{b}}\right)}(t)$ amplitude coefficients are simultaneously nonzero. Such a condition is fulfilled if the two energies are excited simultaneously, which is achieved if the two pulses of $\mathcal{E}_{2}(t)$ overlap in a temporal range. The effect of these interference terms is "dressing" the isolated resonance survival probability $I_{\mathrm{a}}(t)=\left|d_{\mathrm{a}}^{\left(E_{\mathrm{a}}\right)}(t)\right|^{2}$, and therefore changing its shape. As a result of this change in shape, the lifetime associated with $I_{\mathrm{a}}(t)$ also changes from $\tau_{\mathrm{a}}^{\text {iso }}$ to a different value. By varying the delay time $\Delta t$ between the pulses, one can modify the range of temporal overlap between them, and therefore the relative magnitude of the $d_{\mathrm{a}}^{\left(E_{\mathrm{a}}\right)}(t)$ and $d_{\mathrm{a}}^{\left(E_{\mathrm{b}}\right)}(t)$ amplitudes excited simultaneously. This causes variation of the interference terms in a controlled manner, which leads to a change in the shape of $I_{\mathrm{a}}(t)$ and to the control of the associated lifetime.

As shown by eqn (8), the basis of the resonance lifetime control is the quantum interference between resonance amplitudes generated simultaneously in the interaction region of $\hat{H}$ by different pulses of the laser field applied, with a delay time between them. Clearly, this interference is not possible if the amplitudes are not generated at the same time, and the interference effect vanishes when the resonance population decays completely. Thus the requirement of simultaneous population of the interfering amplitudes implies a temporal overlap of the pulses to a certain extent, which means that the delay time between them must be smaller than the sum of their half temporal widths. It is noted from the above equations that the quantum interference on which the control effect is based does not require particularly high amplitudes of the pulses $\left(A_{1}\right.$ and $\left.A_{2}\right)$, which allows application of the control scheme under weak-field consditions.

\subsection{Control of the asymptotic fragment state distribution}

The fragment state distribution produced upon resonance decay is the other property that determines the outcome of a resonancemediated molecular process. The final fragment distribution of a molecular system at a given energy $E$ is determined by the asymptotic behavior - in the region of separated fragments - of the corresponding stationary eigenfunction $\psi_{E}$, which is an intrinsic property of $\hat{H}$. However, despite this intrinsic character, it is also possible to modify and control the energy-resolved asymptotic fragment distribution produced upon resonance decay by means of quantum interference in the weak-field regime, as shown in the following. ${ }^{44}$

The asymptotic probability of the fragment state $\varphi_{E, m}$ at energy $E$ can be expressed as

$$
P_{m}\left(E, t_{\infty}\right)=\mathcal{C} \lim _{t \rightarrow \infty}\left|\left\langle\varphi_{E, m} \mid \Phi(t)\right\rangle\right|^{2}=\mathcal{C}\left|\left\langle\varphi_{E, m} \mid \Phi\left(t_{\infty}\right)\right\rangle\right|^{2},
$$

where $\mathcal{C}$ is a constant and $\Phi(t)$ is the wave packet created by the electric field applied. Let us suppose now that we want to control the energy-resolved asymptotic fragment distribution produced upon decay of the resonance associated with energy $E_{\mathrm{a}}$. To achieve this goal by quantum interference we again apply the two-pulse laser field $\mathcal{E}_{2}(t)$ of eqn (4), that excites the same two resonance energies $E_{\mathrm{a}}$ and $E_{\mathrm{b}}$, and thus creates a wave packet $\Phi(t)$ like that of eqn (5). The only difference with the previous situation of the control of the resonance lifetime is that now the delay time $\Delta t=t_{2}-t_{1}$ between the two pulses is very long. The reason for the long delay time of the second pulse exciting the $E_{\mathrm{b}}$ energy with respect to the first one is to allow enough time for the first resonance excited at $E_{\text {a }}$ to decay completely and to reach the asymptotic time regime of the 
fragment distribution produced. Then, by using the expression of $\Phi(t)$ of eqn (6), where the asymptotic fragment states $\varphi_{E_{\mathrm{a}}, m}$ have been populated after the decay of the population excited to $E_{\text {a }}$, we can project out $\Phi(t)$ onto the fragment states $\varphi_{E_{\mathrm{a}}, m}$ to calculate the distribution at energy $E_{\mathrm{a}}$,

$$
\begin{aligned}
\left\langle\varphi_{E_{\mathrm{a}}, m} \mid \Phi(t)\right\rangle & =\sum_{m^{\prime}} \int \mathrm{d} E^{\prime}\left[b_{E^{\prime}, m^{\prime}}^{\left(E_{\mathrm{a}}\right)}(t)+b_{E^{\prime}, m^{\prime}}^{\left(E_{\mathrm{b}}\right)}(t)\right]\left\langle\varphi_{E_{\mathrm{a}}, m} \mid \varphi_{E^{\prime}, m^{\prime}}\right\rangle \mathrm{e}^{-i E^{\prime} t / \hbar} \\
& =\sum_{m^{\prime}} \int \mathrm{d} E^{\prime}\left[b_{E^{\prime}, m^{\prime}}^{\left(E_{\mathrm{a}}\right)}(t)+b_{E^{\prime}, m^{\prime}}^{\left(E_{\mathrm{b}}\right)}(t)\right] \delta_{m m^{\prime}} \delta\left(E^{\prime}-E_{\mathrm{a}}\right) \mathrm{e}^{-i E^{\prime} t / \hbar} \\
& =\left[b_{E_{\mathrm{a}}, m}^{\left(E_{\mathrm{a}}\right)}(t)+b_{E_{\mathrm{a}}, m}^{\left(E_{\mathrm{b}}\right)}(t)\right] \mathrm{e}^{-i E_{\mathrm{a}} t / \hbar},
\end{aligned}
$$

where eqn (1) were used. The product state distribution at a time $t$ long enough finally becomes

$$
\begin{aligned}
P_{m}\left(E_{\mathrm{a}}, t\right)= & \mathcal{C}\left|\left\langle\varphi_{E_{\mathrm{a}}, m} \mid \Phi(t)\right\rangle\right|^{2}=\mathcal{C}\left[\left|b_{E_{\mathrm{a}}, m}^{\left(E_{\mathrm{a}}\right)}(t)\right|^{2}+b_{E_{\mathrm{a}}, m}^{\left(E_{\mathrm{a}}\right)}(t) b_{E_{\mathrm{a}}, m}^{\left(E_{\mathrm{b}}\right) *}(t)\right. \\
& \left.+b_{E_{\mathrm{a}}, m}^{\left(E_{\mathrm{a}}\right) *}(t) b_{E_{\mathrm{a}}, m}^{\left(E_{\mathrm{b}}\right)}(t)+\left|b_{E_{\mathrm{a}}, m}^{\left(E_{\mathrm{b}}\right)}(t)\right|^{2}\right]
\end{aligned}
$$

Eqn (11) is very similar to eqn (8) in that it also consists of a sum of four terms, three of which arise from the excitation of both the $E_{\mathrm{a}}$ and $E_{\mathrm{b}}$ energies and the interference between them. If a single-pulse $\mathcal{E}_{1}(t)$ field is applied to excite $E_{\mathrm{a}}$, then $\xi_{E_{\mathrm{a}}}(t) \neq 0$, $\xi_{E_{\mathrm{b}}}(t)=0$, and the distribution becomes $P_{m}\left(E_{\mathrm{a}}, t\right)=\mathcal{C}\left|b_{E_{\mathrm{a}}, m}^{\left(E_{\mathrm{a}}\right)}(t)\right|^{2}$, where interference is absent. Excitation of the second energy $E_{\mathrm{b}}$ by applying $\mathcal{E}_{2}(t)$ induces interference between the overlapping resonances which manifests itself in the three additional terms of eqn (11). It is stressed that the interference terms of eqn (11) will appear as long as the amplitude $\xi_{E_{\mathrm{b}}}(t)$ is created by the second pulse of $\mathcal{E}_{2}(t)$, and this can be done at any asymptotic time as long as desired, and as many times as desired (using further successive pulses after the second one in the laser field). ${ }^{44}$

The mechanism of interference between amplitudes produced in continuum fragment states by exciting two overlapping resonances with a long delay time between the two excitations may appear somewhat ellusive. It can be described as follows. Initially the first pulse of $\mathcal{E}_{2}(t)$ excites the resonance energy $E_{\text {a }}$ creating the $\xi_{E_{\mathrm{a}}}(t)$ wave packet amplitude. After a long time, once this amplitude has decayed completely to the continuum states and has reached the asymptotic behavior, the second pulse of $\mathcal{E}_{2}(t)$ excites the resonance energy $E_{\mathrm{b}}$ creating the $\xi_{E_{\mathrm{b}}}(t)$ amplitude. Since both resonances overlap they share common continuum states $\varphi_{E^{\prime}, m^{\prime}}$ in a range of energies $E^{\prime}$. The second pulse initially populates the $\chi_{k}$ states in the interaction region with an amplitude $a_{k}^{\left(E_{\mathrm{b}}\right)}(t)$. After some time this amplitude decays to the continuum states $\varphi_{E^{\prime}, m^{\prime}}$, spreading and redistributing temporarily among those $\varphi_{E^{\prime}, m^{\prime}}$ states which are shared by both $\xi_{E_{\mathrm{a}}}$ and $\xi_{E_{\mathrm{b}}}$, producing the amplitude $b_{E_{\mathrm{a}}, m}^{\left(E_{\mathrm{b}}\right)}(t)$. The appearance of this amplitude is what causes interference with the "asymptotic" $b_{E_{\mathrm{a}}, m}^{\left(E_{\mathrm{a}}\right)}(t)$ amplitude generated much earlier by the decay of the resonance amplitude excited by the first pulse. The amplitude $b_{E_{\mathrm{a}}, m}^{\left(E_{\mathrm{b}}\right)}(t)$, however, is generated in the $\varphi_{E_{\mathrm{a}}, m}$ states only temporarily, during the time that the second delayed pulse excites amplitude to $E_{\mathrm{b}}$ that will further decay initially to $\varphi_{E_{a}, m}$ states shared with $\xi_{E_{\mathrm{a}}}$. After the second pulse is over all the $b_{E_{\mathrm{a}}, m}^{\left(E_{\mathrm{b}}\right)}(t)$ amplitude temporarily generated in $\varphi_{E_{\mathrm{a}}, m}$ states vanishes, redistributing among $\varphi_{E_{\mathrm{b}}, m}$ states that contribute to the asymptotic distribution $P_{m}\left(E_{\mathrm{b}}, t_{\infty}\right)$, because the amplitude excited at $E_{\mathrm{b}}$ cannot contribute to the asymptotic $E_{\mathrm{a}}$ distribution $P_{m}\left(E_{\mathrm{a}}, t_{\infty}\right)$. As a result, the interference effect ceases, and $P_{m}\left(E_{\mathrm{a}}, t_{\infty}\right)$ converges to $P_{m}\left(E_{\mathrm{a}}, t_{\infty}\right)=\mathcal{C}\left|b_{E_{\mathrm{a}}, m}^{\left(E_{\mathrm{a}}\right)}\left(t_{\infty}\right)\right|^{2}$. This is the expected result, in agreement with the previous prediction ${ }^{50}$ that no permanent weak-field coherent control of fragment distributions is possible after the excitation pulse has vanished.

The weak-field strategy applied to control the fragment distribution and reflected in the interference of eqn (11) has similarities with that used in strong-field control techniques. Indeed, the second pulse of $\mathcal{E}_{2}(t)$ plays a similar role as the control field in strong-field techniques, in the sense that it produces the desired control effect while it is on. The difference is that the control effect on the fragment distribution ceases when the control pulse is over in the present weak-field scheme, while it remains in the strong-field case. This, however, does not prevent an effective control of the fragment distribution in the case of the weak-field scheme, if the fragments are detected or moved to other vibronic states of interest (applying a further laser pulse) while the interference effect takes place.

The origin and the basis of the interference in eqn (8) and (11), which govern the control of the resonance lifetime and the fragment diistribution, respectively, are essentially the same, and they only differ in the magnitude of the delay time between the pulses exciting $E_{\mathrm{a}}$ and $E_{\mathrm{b}}$ (relatively short in the case of the lifetime, and very long, in the asymptotic time regime for the fragment distribution). This common origin of the interference effect allows one to design a unified, single control scheme for the two resonance properties. Indeed, a simple way to control both the lifetime and the fragment distribution using a single scheme is to apply two "control pulses", with a different delay time, with each of these pulses controlling one of the two resonance properties. More specifically, the electric field applied would consist of a combination of three Gaussian pulses,

$$
\begin{aligned}
\mathcal{E}_{3}(t)= & A_{1} \mathrm{e}^{-\left(t-t_{1}\right)^{2} / 2 \sigma^{2}} \cos \left[\omega_{1}\left(t-t_{1}\right)+\phi_{1}\right] \\
& +A_{2} \mathrm{e}^{-\left(t-t_{2}\right)^{2} / 2 \sigma^{2}} \cos \left[\omega_{2}\left(t-t_{2}\right)+\phi_{2}\right] \\
& +A_{3} \mathrm{e}^{-\left(t-t_{3}\right)^{2} / 2 \sigma^{2}} \cos \left[\omega_{3}\left(t-t_{3}\right)+\phi_{3}\right],
\end{aligned}
$$

where the first pulse centered at $t_{1}$ would excite the energy $E_{\mathrm{a}}$ of the resonance for which we want to control the lifetime and the fragment distribution, creating the amplitude $\xi_{E_{a}}$; the second pulse centered at $t_{2}$ would excite the energy $E_{\mathrm{b}}$ generating the amplitude $\xi_{E_{\mathrm{b}}}$ with a delay $\Delta t_{12}=t_{2}-t_{1}$ relatively short so as to overlap in time with the first pulse, thus allowing for the control of the resonance lifetime; and the third pulse centered at $t_{3}$ 
would also excite the energy $E_{\mathrm{b}}$ creating $\xi_{E_{\mathrm{b}}}{ }^{\prime}$, but now with a very long delay $\Delta t_{13}=t_{3}-t_{1}$ which allows for the control of the fragment distribution following eqn (11). In the simulations $t_{1}$ is fixed at $t_{1}=0$, which implies that $\Delta t_{12}=t_{2}$ and $\Delta t_{13}=t_{3}$. Thus, application of a single electric field makes possible the control of the two resonance properties that govern a molecular process by just using the appropriate delay time between the different pulses involved in the field.

A few remarks on the above control scheme are now due. In the previous discussion of the equations we have restricted to the excitation of the resonance energies (i.e., the energy of the resonance lineshape peak) for $E_{\mathrm{a}}$ and $E_{\mathrm{b}}$. However, this is not a requirement of the scheme, and any other energies $E_{\mathrm{a}}$ and $E_{\mathrm{b}}$ different from the resonance ones can be excited, as long as the resonances involved overlap at those energies. The only avantage of exciting the resonance energies is that the control scheme becomes more efficient in principle, because the amplitude generated at the lineshape peaks associated with $E_{\mathrm{a}}$ and $E_{\mathrm{b}}$ is maximized (then maximizing the intensity of interference) with respect to other pairs of energies. For simplicity of the above description excitation of only two energies has been considered. But in the scheme the number of energies excited is not restricted, and excitation of more than two energies also increases the efficiency of the interference effect for the control of both the lifetime ${ }^{37}$ and the fragment distribution. ${ }^{44}$ All the above indicates that the applicability of the control scheme is quite flexible and general.

\section{Results and discussion}

In order to illustrate how the unified control scheme works, the simulations will focus on the excitation of the $\mathrm{Ne}-\mathrm{Br}_{2}\left(\mathrm{~B}, \nu^{\prime}\right.$, $n^{\prime}=0$ ) ground intermolecular resonance in two different situations, namely $\nu^{\prime}=27$ and $\nu^{\prime}=35$. The essential difference between them is that the resonances overlap more strongly in the case of $\nu^{\prime}=35$. Regarding the specific parameters of the $\mathcal{E}_{3}(t)$ field applied in the simulations, for simplicity it is assumed that $\phi_{1}=\phi_{2}=\phi_{3}=0$. The amplitudes are $A_{1}=A_{2}=$ $1.0 \times 10^{-6}$ a.u., and $A_{3}=3.0 \times 10^{-6}$ a.u. in the case of $\nu^{\prime}=27$, while for $\nu^{\prime}=35$ the values are $A_{1}=A_{2}=1.0 \times 10^{-6}$ a.u., and $A_{3}=$ $2.0 \times 10^{-6}$ a.u. All these amplitudes correspond to a maximum pulse intensity of the order of $10^{4} \mathrm{~W} \mathrm{~cm}^{-2}$, within the weakfield regime. The same full width at half maximum, $\mathrm{FWHM}=$ $200 \mathrm{ps}$ (related to $\sigma$ ), is used for all the pulses in the different simulations. With this temporal width the corresponding spectral profiles of the pulses of $\mathcal{E}_{3}(t)$ are narrow and do not overlap.

Two electric fields are applied in the simulations, namely $\mathcal{E}_{1}(t)$ and $\mathcal{E}_{3}(t)$, in order to compare the results obtained for both the resonance lifetime and the fragment distribution in the situations of the absence of interference when exciting a single energy, and when two interfering energies are excited. In both cases the survival probability associated with the ground intermolecular resonance of either the $\nu^{\prime}=27$ or $\nu^{\prime}=35$ vibrational manifolds is calculated according to eqn (8) as $I_{k=0}(t)=$ $\left|\left\langle\psi_{k=0} \mid \Phi(t)\right\rangle\right|^{2}$, where $\psi_{k=0}$ is the wave function associated with the ground resonance, and $\Phi(t)$ is the wave packet prepared by the electric field applied. Now the corresponding lifetime, $\tau$, is obtained by fitting $I_{k=0}(t)$ to the function

$$
I_{k=0}\left(t_{j}\right)=A \int_{-\infty}^{t_{j}} \mathrm{CC}(t)\left[\exp \left(-\left(t_{j}-t\right) / \tau\right)\right] \mathrm{d} t,
$$

$\mathrm{CC}(t)$ being the cross-correlation function of the laser field $\mathcal{E}_{1}(t)$ or $\mathcal{E}_{3}(t)$ and $A$ an amplitude scaling parameter.

The energy-resolved product fragment state distribution is calculated as follows. In the simulations the wave packet is represented in Jacobian coordinates $(R, r, \theta)$, where $R$ is the distance between the Ne atom and the $\mathrm{Br}_{2}$ center of mass, $r$ is the $\mathrm{Br}-\mathrm{Br}$ internuclear distance, and $\theta$ is the angle between the vectors associated with $R$ and $r$. In this representation the rovibrational eigenstates associated with the $\mathrm{Br}_{2}(\mathrm{~B}, v, j)$ fragment are $\zeta_{v}^{(j)}(r) P_{j}(\cos \theta)$, where $\zeta_{v}^{(j)}(r)$ are the vibrational eigenfunctions of $\mathrm{Br}_{2}(\mathrm{~B})$ with associated energies $E_{v, j}$ and $P_{j}(\cos \theta)$ are Legendre polynomials, $v$ and $j$ being the $\mathrm{Br}_{2}$ vibrational and rotational quantum numbers, respectively. The population of the $\operatorname{Br}_{2}(\mathrm{~B}, v, j)$ fragment states is computed along time by projecting out the wave packet onto the corresponding states

$$
P_{v, j}(E, t)=C k_{\nu, j}\left|\int_{0}^{t}\left\langle\zeta_{v}^{(j)}(r) P_{j}(\cos \theta) \mid \Phi\left(R_{\mathrm{c}}, r, \theta, t^{\prime}\right)\right\rangle \mathrm{e}^{i E t^{\prime} / \hbar} \mathrm{d} t^{\prime}\right|^{2},
$$

where $C$ is a constant factor, $R_{\mathrm{c}}$ is a suitably large distance of the dissociation coordinate $R, E$ is the total energy of the system, and $k_{v, j}$ is given by

$$
k_{v, j}=\left[2 \mu_{\mathrm{Br}_{2}}\left(E-E_{v, j}\right)\right]^{1 / 2},
$$

with $\mu_{\mathrm{Br}_{2}}$ being the $\mathrm{Br}_{2}$ reduced mass. The population in each vibrational state of $\mathrm{Br}_{2}(\mathrm{~B}, v)$ is now calculated as

$$
P_{v}(E, t)=\sum_{j} P_{v, j}(E, t) .
$$

In the first set of simulations the $\mathrm{Ne}-\mathrm{Br}_{2}\left(\mathrm{~B}, \nu^{\prime}=27, n^{\prime}=0\right)$ ground intermolecular resonance is excited. As shown in the associated excitation spectrum (Fig. 1), this resonance overlaps with some orbiting resonances of $\mathrm{Ne}-\mathrm{Br}_{2}\left(\mathrm{~B}, \nu=\nu^{\prime}-1=26\right){ }^{5,35}$ The main peak of the spectrum located at $E_{\mathrm{a}}=-61.8 \mathrm{~cm}^{-1}$ corresponds to the $\mathrm{Ne}-\mathrm{Br}_{2}\left(\mathrm{~B}, \nu^{\prime}=27, n^{\prime}=0\right)$ ground resonance, while the peak at $E_{\mathrm{b}}=-60.63 \mathrm{~cm}^{-1}$ and a weak peak at $E=-59.5 \mathrm{~cm}^{-1}$ correspond to two $v=26$ orbiting resonances overlapping with each other and with the $\nu^{\prime}=27$ ground resonance. The spectral profiles of the three pulses of $\mathcal{E}_{3}(t)\left(A_{1}=A_{2}, A_{3}=3 A_{1}\right.$; the first pulse of $\mathcal{E}_{3}(t)$ coincides with that of $\left.\mathcal{E}_{1}(t)\right)$ are plotted in Fig. 1. As shown in the figure, there is no spectral overlap between the first pulse of $\mathcal{E}_{3}(t)$ and the other two.

As discussed above, when the single-pulse field $\mathcal{E}_{1}(t)$ is applied to excite only the energy $E_{\mathrm{a}}$, there is no possibility of interference and the asymptotic fragment distribution becomes $P_{m}\left(E_{\mathrm{a}}, t_{\infty}\right)=\mathcal{C}\left|b_{E_{\mathrm{a}}, m}^{\left(E_{\mathrm{a}}\right)}\left(t_{\infty}\right)\right|^{2}$. In the case of $\mathrm{Ne}-\mathrm{Br}_{2}\left(\mathrm{~B}, \nu^{\prime}=27\right)$, when the energy $E_{\mathrm{a}}=-61.8 \mathrm{~cm}^{-1}$ is excited by $\mathcal{E}_{1}(t)$, the energyresolved $\mathrm{Br}_{2}\left(\mathrm{~B}, v_{\mathrm{f}}\right)$ vibrational distribution associated with $E_{\mathrm{a}}$, calculated with eqn (14)-(16), is shown in Fig. 2. The population 


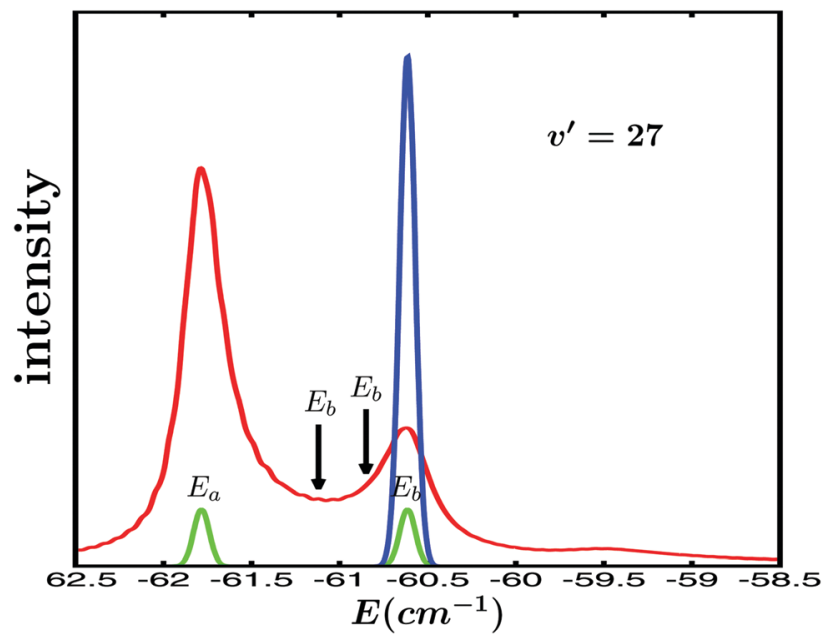

Fig. 1 Excitation spectrum of the $\mathrm{Ne}-\mathrm{Br}_{2}\left(\mathrm{~B}, \nu^{\prime}=27\right)$ ground intermolecular resonance. The energies $E_{\mathrm{a}}=-61.8 \mathrm{~cm}^{-1}$ and $E_{\mathrm{b}}=-60.63 \mathrm{~cm}^{-1}$ (relative to the $\mathrm{Ne}+\mathrm{Br}_{2}\left(\mathrm{~B}, \nu^{\prime}=27, j^{\prime}=0\right)$ dissociation threshold $)$ of the two overlapping resonances excited by the laser fields are indicated in the figure. Two additional energies $E_{\mathrm{b}}=-60.85 \mathrm{~cm}^{-1}$ and $E_{\mathrm{b}}=-61.12 \mathrm{~cm}^{-1}$ used in the simulations are indicated by arrows. The spectral profiles of the pulses used in the $\mathcal{E}_{1}(t)$ and $\mathcal{E}_{3}(t)$ fields to excite the $E_{\mathrm{a}}$ and $E_{\mathrm{b}}$ energies are also shown

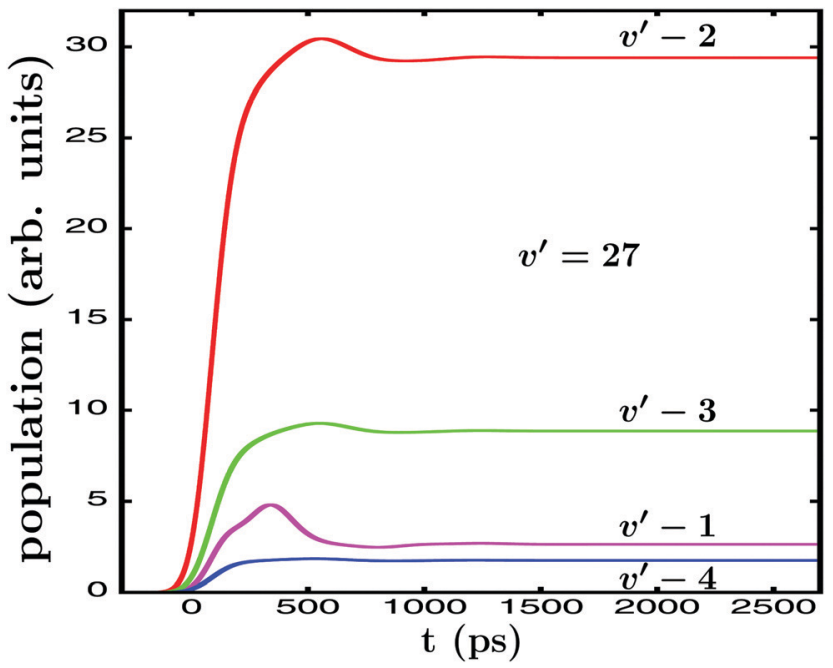

Fig. 2 Energy-resolved $\mathrm{Br}_{2}\left(\mathrm{~B}, v_{\mathrm{f}}\right)$ fragment populations in the $\nu_{\mathrm{f}}=$ $\nu^{\prime}-1, \ldots, \nu^{\prime}-4$ final vibrational state, upon predissociation of $\mathrm{Ne}-\mathrm{Br}_{2}(\mathrm{~B}$, $\left.\nu^{\prime}=27\right)$ when the $E_{\mathrm{a}}-61.8 \mathrm{~cm}^{-1}$ energy is excited by the single-pulse $\mathcal{E}_{1}(t)$ field.

distributes among four vibrational final states, $\nu_{\mathrm{f}}=\nu^{\prime}-1, \nu^{\prime}-2$, $\nu^{\prime}-3$, and $\nu^{\prime}-4$, and these populations reach their asymptotic, constant values for $t>1000 \mathrm{ps}$.

Now, in order to exert control on both the $\mathrm{Ne}-\mathrm{Br}_{2}\left(\mathrm{~B}, \nu^{\prime}=27\right)$ ground resonance lifetime and the associated fragment distribution, the $\mathcal{E}_{3}(t)$ field of eqn (12) is applied. The first and second pulses (centered at $t_{1}$ and $t_{2}$, respectively) excite the $E_{\mathrm{a}}$ and $E_{\mathrm{b}}$ energies, respectively, with a delay time $\Delta t_{12}=160 \mathrm{ps}$. The reason for choosing this specific delay time $\Delta t_{12}$ is because it was previously shown that it maximizes the lifetime of the $\mathrm{Ne}-\mathrm{Br}_{2}\left(\mathrm{~B}, \nu^{\prime}=27\right)$ ground resonance. ${ }^{36}$ With such a delay time the first and second pulses of $\mathcal{E}_{3}(t)$ overlap in time to some extent, as shown by the $\mathcal{E}_{3}(t)$ temporal profile of Fig. 3(a). The next step is the modification of the $\mathrm{Br}_{2}\left(\mathrm{~B}, v_{\mathrm{f}}\right)$ fragment vibrational distribution, by applying the third pulse (centered at $t_{3}$ ) of $\mathcal{E}_{3}(t)$ to excite again the $E_{\mathrm{b}}$ energy, but with a long delay time $\Delta t_{13}$ within the asymptotic time region $t>1000$ ps of the distribution. Specifically, two values $\Delta t_{13}=1500$ and $2200 \mathrm{ps}$ have been chosen. The reason for using two different $\Delta t_{13}$ values instead of only one is to illustrate the fact that the interference effect

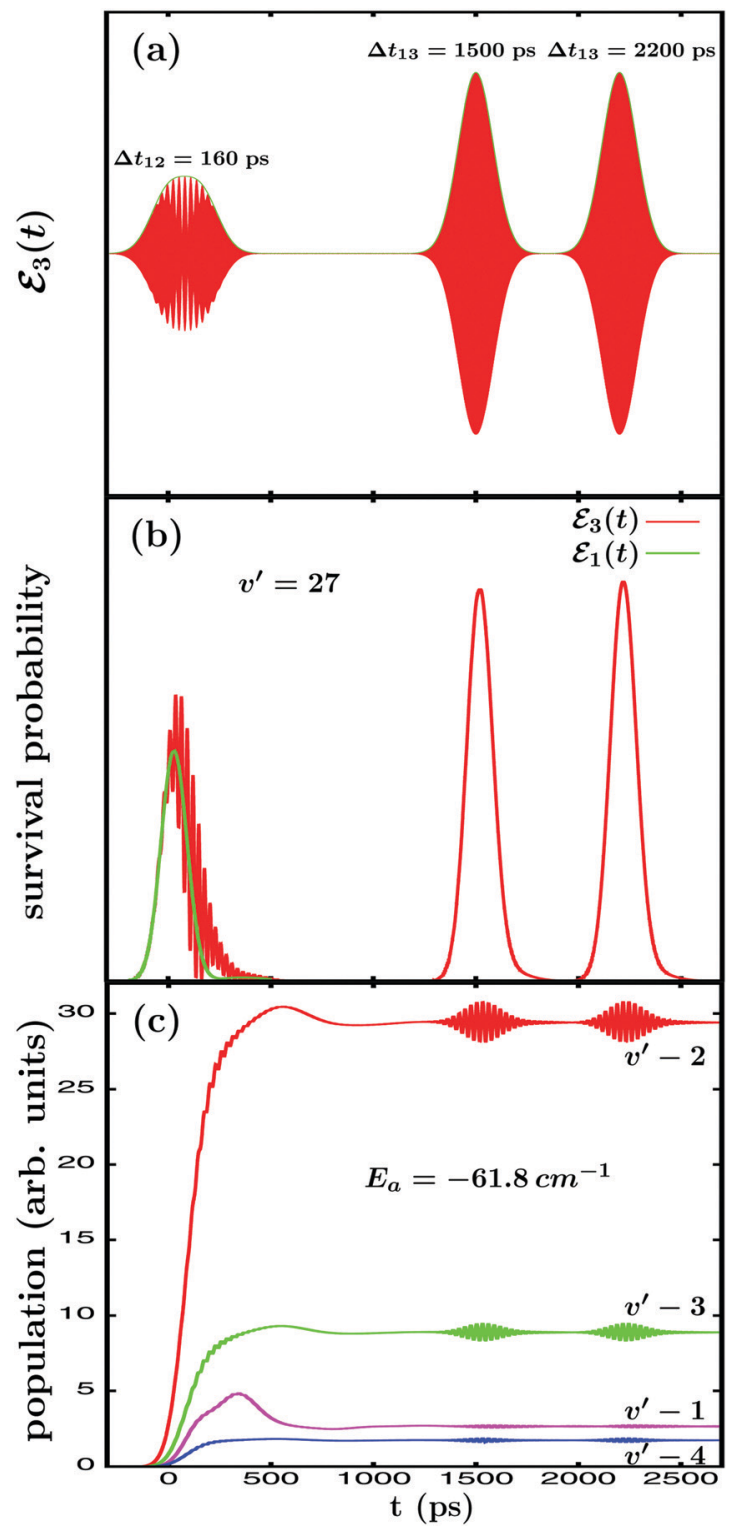

Fig. 3 (a) Temporal profile of the $\mathcal{E}_{3}(t)$ laser field applied in the case of $\mathrm{Ne}-\mathrm{Br}_{2}\left(\mathrm{~B}, \nu^{\prime}=27\right)$. While $\Delta t_{12}=160 \mathrm{ps}$, two situations are shown where the delay time $\Delta t_{13}=t_{3}-t_{1}$ of the third pulse of $\mathcal{E}_{3}(t)$ takes two different values, namely $\Delta t_{13}=1500$ and 2200 ps. (b) Survival probability of the $\mathrm{Ne}-\mathrm{Br}_{2}(\mathrm{~B}$, $\left.\nu^{\prime}=27\right)$ ground intermolecular resonance calculated when the singlepulse $\mathcal{E}_{1}(t)$ and the $\mathcal{E}_{3}(t)$ electric fields are applied. (c) Energy-resolved $\mathrm{Br}_{2}\left(\mathrm{~B}, v_{\mathrm{f}}\right)$ fragment vibrational populations in the $\nu_{\mathrm{f}}=\nu^{\prime}-1, \ldots, \nu^{\prime}-4$ final vibrational state associated with the $E_{\mathrm{a}}=-61.8 \mathrm{~cm}^{-1}$ energy, produced upon predissociation of $\mathrm{Ne}-\mathrm{Br}_{2}\left(\mathrm{~B}, \nu^{\prime}=27\right)$ when the $\mathcal{E}_{3}(t)$ field is applied, with two delay times, $\Delta t_{13}=1500$ and $2200 \mathrm{ps}$, between the first and third pulses of $\mathcal{E}_{3}(t)$. The energy $E_{\mathrm{b}}=-60.85 \mathrm{~cm}^{-1}$ is excited by $\mathcal{E}_{3}(t)$. 
can be induced in the asymptotic fragment distribution as many times as desired, as discussed above. The whole temporal profiles of the $\mathcal{E}_{3}(t)$ fields applied with the two $\Delta t_{13}$ delays are shown in Fig. 3(a).

The survival probability of the $\mathrm{Ne}-\mathrm{Br}_{2}\left(\mathrm{~B}, \nu^{\prime}=27\right)$ ground resonance, $I_{k=0}(t)$, calculated when the single-pulse field $\mathcal{E}_{1}(t)$ and the $\mathcal{E}_{3}(t)$ fields of Fig. 3(a) are applied are displayed in Fig. 3(b). When $\mathcal{E}_{1}(t)$ is used, $I_{k=0}(t)=\left|d_{k=0}^{\left(E_{\mathrm{a}}\right)}(t)\right|^{2}$ displays the shape of the convolution of the Gaussian pulse crosscorrelation curve with the exponential decay associated with a single resonance, reflected by eqn (13). By fitting $I_{k=0}(t)$ with eqn (13) the associated lifetime $\tau_{k=0}^{\text {iso }}=23.5 \mathrm{ps}$ is obtained. ${ }^{36}$ By applying $\mathcal{E}_{3}(t)$ the $E_{\mathrm{a}}$ and $E_{\mathrm{b}}$ energies are excited by the first two pulses, and they interfere. This interference manifests itself in pronounced undulations in $I_{k=0}(t)$ in the time range where the two interfering resonances remain populated. This is the interference effect reflected by eqn (8). As previously shown, ${ }^{36}$ those undulations are separated by a constant time interval which is the inverse of the energy difference $E_{\mathrm{b}}-E_{\mathrm{a}}$. By fitting now this $I_{k=0}(t)$ function with eqn (13) a lifetime $\tau_{k=0}=75$ ps is obtained. ${ }^{36}$ Thus an enhancement of the resonance lifetime by a factor of three can be achieved by means of quantum interference. The survival probability of Fig. 3(b) shows that when the third pulse of $\mathcal{E}_{3}(t)$ is applied to excite $E_{\mathrm{b}}$ with the two $\Delta t_{13}$ delays, the ground resonance is populated again, which is the behavior expected for resonances that overlap at different energies, among them $E_{\mathrm{b}}$.

The $\mathrm{Br}_{2}\left(\mathrm{~B}, \nu_{\mathrm{f}}<\nu^{\prime}\right)$ fragment distributions obtained when the $\mathcal{E}_{3}(t)$ fields of Fig. 3(a) are applied are shown in Fig. 3(c). A clear modification of the asymptotic vibrational populations is induced when the third pulse of $\mathcal{E}_{3}(t)$ excites the $E_{\mathrm{b}}$ energy at the two $\Delta t_{13}$ delays, as previously found. ${ }^{44}$ During the application of the third pulse the populations display some undulations that reflect the interference effect predicted by eqn (11). As discussed above, once the third pulse is over and the resonance excited at $E_{\mathrm{b}}$ has decayed completely, the asymptotic populations converge back to the values $P_{v_{\mathrm{f}}}\left(E_{\mathrm{a}}, t\right)=\mathcal{C}\left|b_{E_{\mathrm{a}}, v_{\mathrm{f}}}^{\left(E_{\mathrm{a}}\right)}(t)\right|^{2}$ of the populations of Fig. 2. The interference effect is increasingly more intense as the vibrational population is larger in magnitude, because the larger is the amplitude $b_{E_{\mathrm{a}}, v_{\mathrm{f}}}^{\left(E_{\mathrm{f}}\right)}(t)$ the larger will be the interference terms of eqn (11). In this sense it is noted that the second pulse of $\mathcal{E}_{3}(t)$ also causes an interference effect on the vibrational populations of Fig. 3(c) around $\Delta t_{12}=160 \mathrm{ps,} \mathrm{although} \mathrm{much}$ weaker since $A_{2}=A_{3} / 3$.

The results of Fig. 3 provide a practical illustration of the predictions of eqn (8) and (11). They demostrate that it is indeed possible to design a unified control scheme to modify the two resonance properties, namely the lifetime and the fragment distribution produced upon resonance decay, which govern a resonance-mediated molecular process. The above fact remains valid when we move to a stronger overlapping regime, as shown in the following.

The excitation spectrum associated with the $\mathrm{Ne}-\mathrm{Br}_{2}\left(\mathrm{~B}, \nu^{\prime}=\right.$ $35, n^{\prime}=0$ ) ground intermolecular resonance is shown in Fig. 4 .

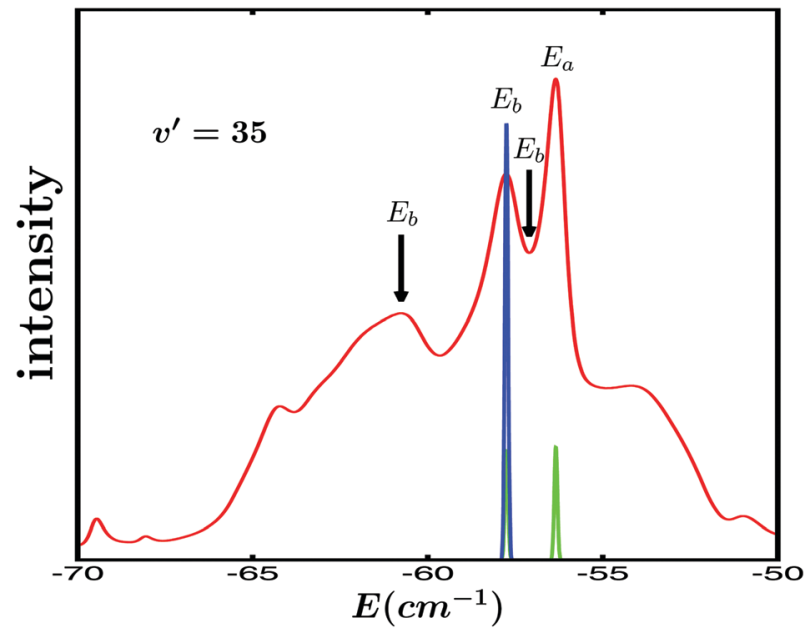

Fig. 4 Excitation spectrum of the $\mathrm{Ne}-\mathrm{Br}_{2}\left(\mathrm{~B}, \nu^{\prime}=35\right)$ ground intermolecular resonance. The energies $E_{\mathrm{a}}=-56.34 \mathrm{~cm}^{-1}$ and $E_{\mathrm{b}}=-57.75 \mathrm{~cm}^{-1}$ (relative to the $\mathrm{Ne}+\mathrm{Br}_{2}\left(\mathrm{~B}, \nu^{\prime}=35, j^{\prime}=0\right)$ dissociation threshold) excited by the laser fields are indicated in the figure. Two additional energies $E_{\mathrm{b}}=$ $-57.10 \mathrm{~cm}^{-1}$ and $E_{\mathrm{b}}=-60.76 \mathrm{~cm}^{-1}$ used in the simulations are indicated by arrows. The spectral profiles of the pulses used in the $\mathcal{E}_{1}(t)$ and $\mathcal{E}_{3}(t)$ fields to excite the $E_{\mathrm{a}}$ and $E_{\mathrm{b}}$ energies are also shown.

It displays a stronger, more congested overlapping regime between different intermolecular resonances. The main peak of the spectrum, located at energy $E_{\mathrm{a}}=-56.34 \mathrm{~cm}^{-1}$, corresponds to the $\nu^{\prime}=35$ ground resonance. This resonance lies below the $\mathrm{Ne}+\mathrm{Br}_{2}\left(\mathrm{~B}, \nu^{\prime}-1=34, j^{\prime}=0\right)$ dissociation threshold, and therefore it is embedded in the spectrum of intermolecular resonances of the $\nu^{\prime}-1=34$ vibrational manifold, which allows the remaining features of the spectrum of Fig. 4 to originate. ${ }^{51}$ Those resonances are broad enough so as to produce a stronger overlapping regime than in the case of $\mathrm{Ne}-\mathrm{Br}_{2}\left(\mathrm{~B}, \nu^{\prime}=27\right)$.

In a first simulation the energy $E_{\mathrm{b}}$ of the spectrum of Fig. 4 chosen to induce interference is $E_{\mathrm{b}}=-57.75 \mathrm{~cm}^{-1}$. While in a strong overlapping regime like that of Fig. 4 several resonances of the $\nu^{\prime}-1=34$ manifold (along with the ground resonance of $\nu^{\prime}=35$ ) overlap at this energy, it was previously determined that among them the dominant resonance is $n=9$. The same as in Fig. 1, the spectral profiles of the three pulses of $\mathcal{E}_{3}(t)\left(A_{1}=A_{2}\right.$, $A_{3}=2 A_{1}$ ) are plotted in Fig. 4 .

In the case of the control of the behavior of the $\mathrm{Ne}-\mathrm{Br}_{2}(\mathrm{~B}$, $\left.\nu^{\prime}=35\right)$ ground resonance, an $\mathcal{E}_{3}(t)$ laser field with a $\Delta t_{12}=$ 120 ps delay time has been applied (see Fig. 5(a)). As found previously, ${ }^{39}$ this is the delay time that maximizes the enhancement of the $\nu^{\prime}=35$ ground resonance lifetime. As in the previous case of $\mathrm{Ne}-\mathrm{Br}_{2}\left(\mathrm{~B}, \nu^{\prime}=27\right)$, the same $\Delta t_{13}$ delays, namely $\Delta t_{13}=1500$ and $2200 \mathrm{ps}$, are used.

The survival probabilities associated with the $\mathrm{Ne}-\mathrm{Br}_{2}(\mathrm{~B}$, $\nu^{\prime}=35, n^{\prime}=0$ ) resonance when the $\mathcal{E}_{1}(t)$ and $\mathcal{E}_{3}(t)$ fields are applied are shown in Fig. 5(b). A qualitatively similar interference pattern to that of Fig. 3(b) is found in $I_{k=0}(t)$ when the $\mathcal{E}_{3}(t)$ field is applied, as compared to the plain survival probability curve obtained with $\mathcal{E}_{1}(t)$. However, the intensity of interference and the change of the shape of $I_{k=0}(t)$ appear to be more pronounced in the present case than for $\nu^{\prime}=27$. Actually, the $\tau_{k=0}^{\text {iso }}$ lifetime 


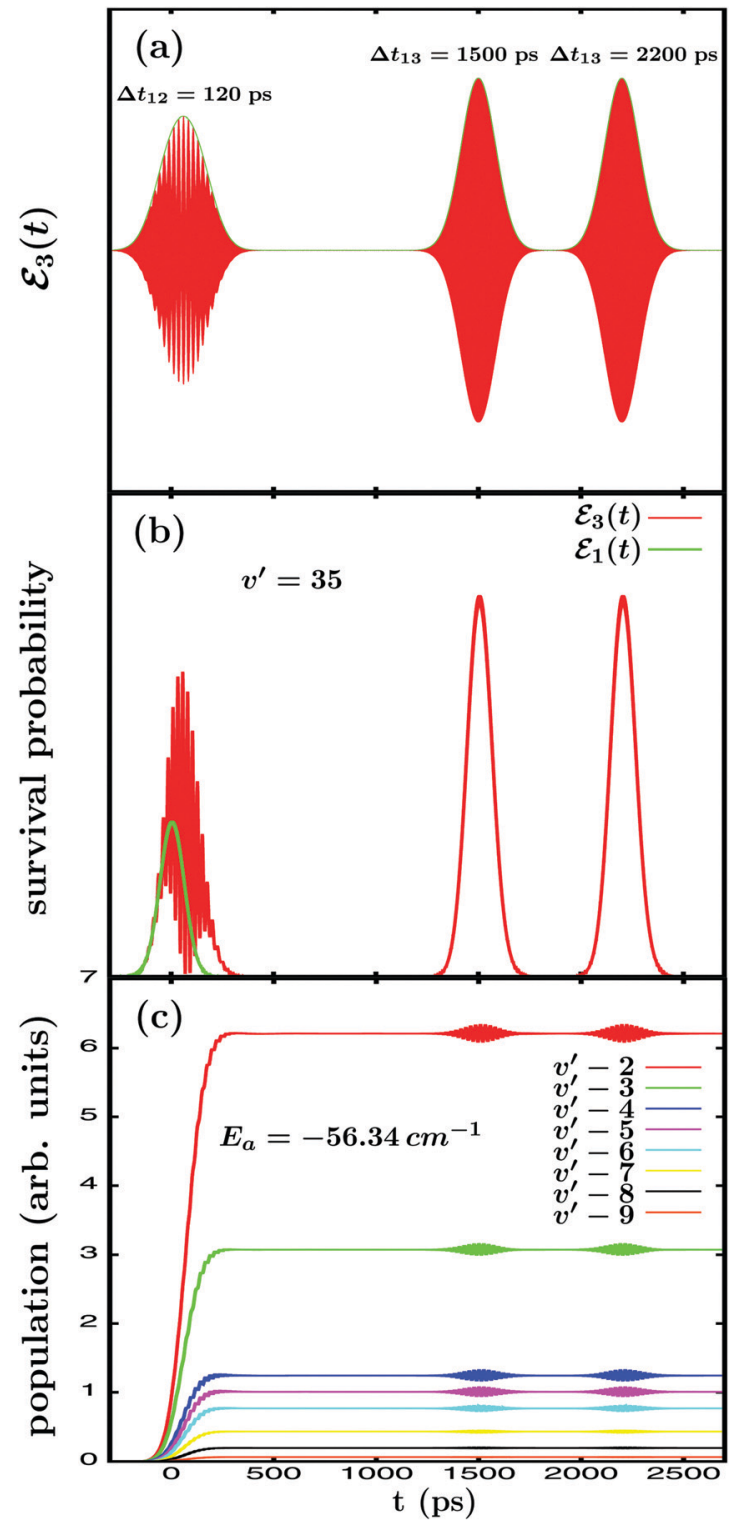

Fig. 5 The same as Fig. 3 but for $\mathrm{Ne}-\mathrm{Br}_{2}\left(\mathrm{~B}, \nu^{\prime}=35\right)$, with $E_{\mathrm{a}}=-56.34 \mathrm{~cm}^{-1}$ and $E_{\mathrm{b}}=-57.75 \mathrm{~cm}^{-1}$.

associated with the survival probability obtained with $\mathcal{E}_{1}(t)$ is $\tau_{k=0}^{\text {iso }}=3.8 \mathrm{ps}$, while the lifetime found when applying $\mathcal{E}_{3}(t)$ is $\tau_{k=0}=$ 61.0 ps. $^{37,39}$ Thus the $\nu^{\prime}=35$ ground resonance lifetime is enhanced by a factor of 15 , compared to the factor of 3 obtained for $\nu^{\prime}=27$. This larger effect of interference in the lifetime enhancement is likely due to the stronger overlapping between the $n^{\prime}=0$ and the $n=9$ resonances compared to the $\nu^{\prime}=27$ situation of Fig. 1. As discussed below, this result is related to the magnitude of the coefficients $C_{E^{\prime}}^{\left(E_{\mathrm{a}}\right)}(t)$ and $C_{E^{\prime}}^{\left(E_{\mathrm{b}}\right)}(t)$ of eqn (8), which depend on the intensity of the excitation spectrum at $E_{\mathrm{a}}$ and $E_{\mathrm{b}}$, which is expected to be higher for stronger resonance overlap. As the overlap between the resonances increases, the magnitude of the amplitudes $d_{k=0}^{\left(E_{\mathrm{a}}\right)}(t)$ and $d_{k=0}^{\left(E_{\mathrm{b}}\right)}(t)$ in eqn (8) increases as well, producing a more intense interference effect. This is supported by the result that when the pair of resonances excited is $n^{\prime}=0$ and $n=7$ (located at $-60.76 \mathrm{~cm}^{-1}$ in the spectrum of Fig. 4), with a weaker overlap than $n^{\prime}=0$ and $n=9, \tau_{k=0}$ was enhanced only up to $\tau_{k=0}=37.0 \mathrm{ps}$ under the same laser field conditions, i.e., somewhat more than half the enhancement achieved with the $n^{\prime}=0$ and $n=9$ resonances. ${ }^{39}$ Thus the main effect of increasing the intensity of resonance overlap would be to enhance the degree of control of the resonance lifetime.

The energy-resolved $\mathrm{Br}_{2}\left(\mathrm{~B}, \nu_{\mathrm{f}}<\nu^{\prime}=35\right)$ fragment distributions obtained by applying the $\mathcal{E}_{3}(t)$ fields of Fig. 5(a) are displayed in Fig. 5(c). Since the $\mathrm{Ne}-\mathrm{Br}_{2}\left(\mathrm{~B}, \nu^{\prime}=35, n^{\prime}=0\right)$ resonance lies below the $\mathrm{Ne}+\mathrm{Br}_{2}\left(\mathrm{~B}, \nu^{\prime}-1=34, j^{\prime}=0\right)$ dissociation threshold, the $\nu^{\prime}-1$ dissociation channel is closed, and the only open channels are $\nu_{\mathrm{f}} \leq \nu^{\prime}-2$. For $\nu^{\prime}=35$ there are twice the number of open channels than for $\nu^{\prime}=27$. The vibrational populations reach their asymptotic, constant values for $t>500 \mathrm{ps}$. The interesting result is that a similar interference effect to that of the distributions of Fig. 3(c) is found in the present distributions when the third pulse of $\mathcal{E}_{3}(t)$ is applied. So again the same qualitative interference behavior is found regardless of the intensity of resonance overlap. In Fig. 5(c) the intensity of the interference effect is somewhat weaker than in Fig. 3(c), which is not surprising since now $A_{2}=2 A_{1}$, while for $\nu^{\prime}=27 A_{2}=3 A_{1}$. However, the same as with the resonance lifetime, the stronger resonance overlap is also expected to favor the intensity of interference in the asymptotic fragment distribution. Indeed, again related to the $C_{E^{\prime \prime}}^{\left(E_{\mathrm{a}}\right)}(t)$ and $C_{E^{\prime \prime}}^{\left(E_{\mathrm{b}}\right)}(t)$ coefficients of eqn (6), the interfering amplitudes $b_{E_{\mathrm{a}}, v_{\mathrm{f}}}^{\left(E_{\mathrm{a}}\right)}(t)$ and $b_{E_{\mathrm{a}}, v_{\mathrm{f}}}^{\left(E_{\mathrm{b}}\right)}(t)$ of eqn (11) will be correspondingly larger for a stronger resonance overlap.

\subsection{Dependence of the control of the $E_{b}$ energy}

In the results of Fig. 3 and 5 discussed so far both the $E_{\mathrm{a}}$ and $E_{\mathrm{b}}$ energies chosen to interfere coincide with resonance energies. As commented above, this is not a requirement of the control scheme, and control is also achieved if different energies are excited by the pump laser field. In fact, exciting $E_{\mathrm{b}}$ energies different from the resonance ones $E_{\mathrm{b}}=-60.63 \mathrm{~cm}^{-1}$ (for $\left.\nu^{\prime}=27\right)$ and $E_{\mathrm{b}}=-57.75 \mathrm{~cm}^{-1}$ (for $\nu^{\prime}=35$ ) illustrates very nicely how the control scheme operates. In this sense, simulations exciting two additional $E_{\mathrm{b}}$ energies different from $E_{\mathrm{b}}=-60.63 \mathrm{~cm}^{-1}$ or $E_{\mathrm{b}}=-57.75 \mathrm{~cm}^{-1}$ in the cases of $\nu^{\prime}=27$ and $\nu^{\prime}=35$, respectively, have been carried out. More specifically, the simulations apply the same $\mathcal{E}_{3}(t)$ laser fields of Fig. 3(a) and 5(a), exciting the same $E_{\mathrm{a}}$ resonance energies as before (i.e., $E_{\mathrm{a}}=61.8 \mathrm{~cm}^{-1}$ for $\nu^{\prime}=27$ and $E_{\mathrm{a}}=56.34 \mathrm{~cm}^{-1}$ for $\left.\nu^{\prime}=35\right)$, but now changing $E_{\mathrm{b}}$ to values of 61.12 and $60.85 \mathrm{~cm}^{-1}$ for $\nu^{\prime}=27$, and 57.10 and $60.76 \mathrm{~cm}^{-1}$ for $\nu^{\prime}=35$. The additional $E_{\mathrm{b}}$ energies excited are indicated by arrows in Fig. 1 and 4 for $\nu^{\prime}=27$ and 35, respectively. Survival probabilities and energy-resolved $\mathrm{Br}_{2}\left(\mathrm{~B}, v_{\mathrm{f}}\right)$ fragment distributions are calculated with the new $E_{\mathrm{b}}$ energies, and they are compared in Fig. 6 and 7 with those shown in Fig. 3 and 5 .

The $I_{k=0}(t)$ survival probabilities found for the three different $E_{\mathrm{b}}$ energies for $\nu^{\prime}=27$ (Fig. 6(a)) and $\nu^{\prime}=35$ (Fig. 7(a)) indeed show an interference pattern in the temporal region where the first and second pulses of $\mathcal{E}_{3}(t)$ overlap, indicating that 
resonance lifetime control is achieved in all cases. However, a clear trend - which is the same for $\nu^{\prime}=27$ and 35 - is found for the intensity of interference when the $E_{\mathrm{b}}$ energy changes: the interference intensity decreases remarkably as the corresponding excitation spectrum intensity (see Fig. 1 and 4) associated with the specific $E_{\mathrm{b}}$ energy decreases. The same effect is also displayed in the survival probability peaks appearing at $\Delta t_{13}=1500$ and 2200 ps. The maximum interference intensity occurs for the resonance energies $E_{\mathrm{b}}=-60.63 \mathrm{~cm}^{-1}$ and $E_{\mathrm{b}}=-57.75 \mathrm{~cm}^{-1}$, which have associated the maximum intensity in the spectra of Fig. 1 and 4, respectively. The interference effect decreases for the other two values of $E_{\mathrm{b}}$ proportionally to the associated decrease in the spectrum intensity.

The explanation of the trend found in Fig. 6(a) and 7(a) for the interference intensity is provided by eqn (6) and (8), and more specifically by the amplitude coefficients $C_{E^{\prime}}^{\left(E_{\mathrm{b}}\right)}(t)$ of eqn (8). Such coefficients denote the amplitude excited by the second (and third) pulse of $\mathcal{E}_{3}(t)$ at energy $E_{\mathrm{b}}$. This amplitude will depend on both the pulse amplitude $A_{2}$ (or $A_{3}$ ) and the spectrum intensity at $E_{\mathrm{b}}$, which in turn is the sum of all the

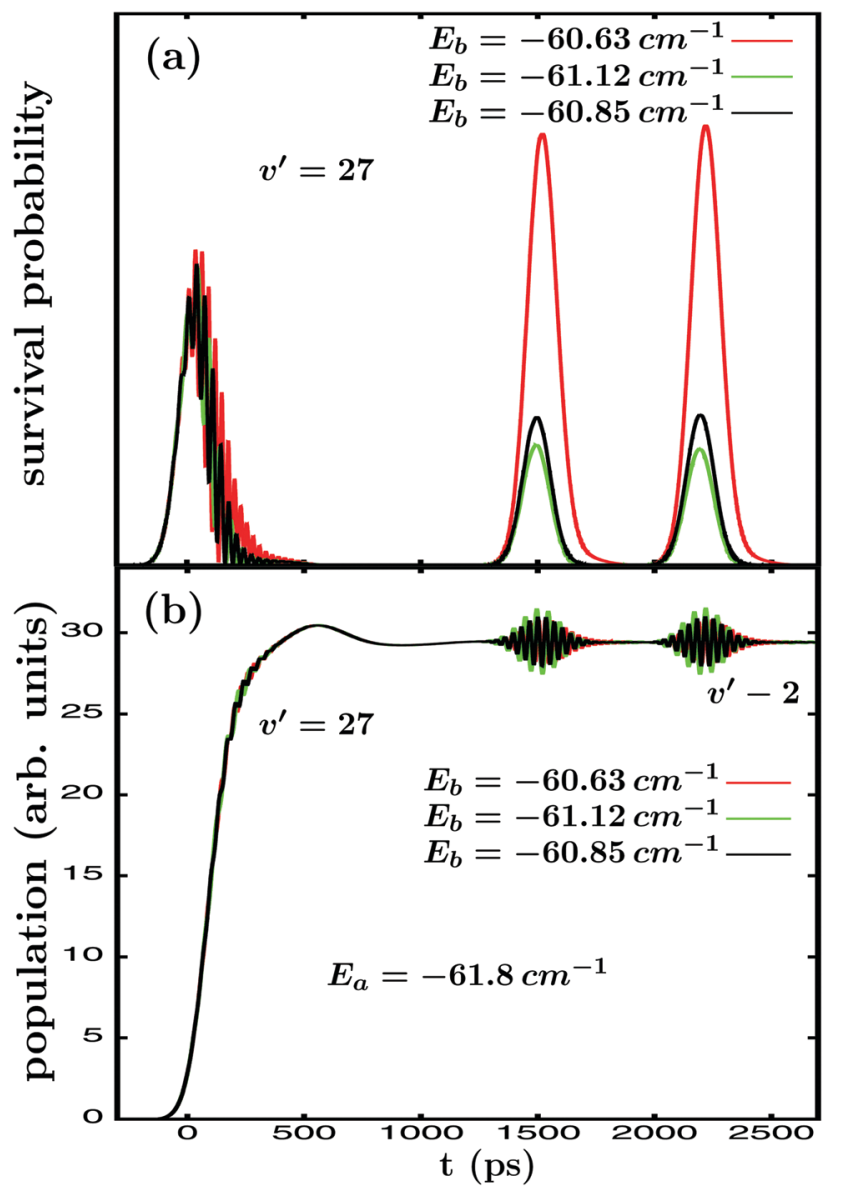

Fig. 6 Comparison of the (a) survival probabilities of the $\mathrm{Ne}-\mathrm{Br}_{2}(\mathrm{~B}$, $\left.\nu^{\prime}=27\right)$ ground intermolecular resonance and (b) energy-resolved $\mathrm{Br}_{2}\left(\mathrm{~B}, v_{\mathrm{f}}\right)$ fragment vibrational populations in the $\nu_{\mathrm{f}}=\nu^{\prime}-2$ final vibrational state associated with the $E_{\mathrm{a}}=-61.8 \mathrm{~cm}^{-1}$ energy, when three different $E_{\mathrm{b}}$ energies, $E_{\mathrm{b}}=-60.63,-61.12$ and $-60.85 \mathrm{~cm}^{-1}$, are excited by applying the $\mathcal{E}_{3}(t)$ electric fields of Fig. 3(a).

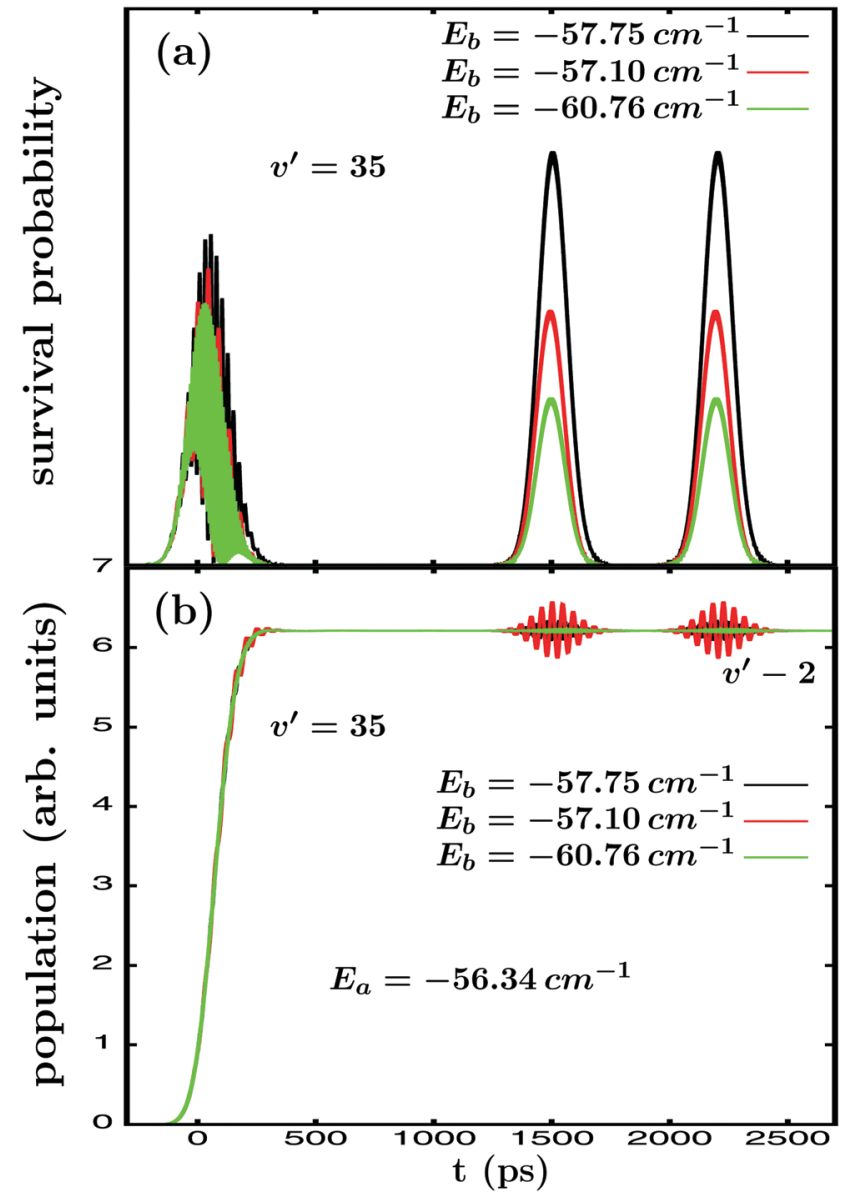

Fig. 7 Comparison of the (a) survival probabilities of the $\mathrm{Ne}-\mathrm{Br}_{2}(\mathrm{~B}$, $\left.\nu^{\prime}=35\right)$ ground intermolecular resonance and (b) energy-resolved $\mathrm{Br}_{2}\left(\mathrm{~B}, v_{\mathrm{f}}\right)$ fragment vibrational population in the $\nu_{\mathrm{f}}=\nu^{\prime}-2$ final vibrational state associated with the $E_{\mathrm{a}}=-56.34 \mathrm{~cm}^{-1}$ energy, when three different $E_{\mathrm{b}}$ energies, $E_{\mathrm{b}}=-57.75,-57.10$ and $-60.76 \mathrm{~cm}^{-1}$, are excited by applying the $\mathcal{E}_{3}(t)$ electric fields of Fig. 5(a).

contributions of the different overlapping resonances at that energy. If $A_{2}$ (or $A_{3}$ ) does not change - since the $\mathcal{E}_{3}(t)$ field is the same in the three simulations of Fig. 6 and $7-C_{E^{\prime}}^{\left(E_{\mathrm{b}}\right)}(t)$ only changes due to the change of spectrum intensity when $E_{\mathrm{b}}$ is varied, and this variation affects the intensity of the interference terms of eqn (8). The implication is that the change in shape of $I_{k=0}(t)$ is smaller for the two additional $E_{\mathrm{b}}$ energies for $\nu^{\prime}=27$ and 35 , which is reflected in a lower enhancement of the ground resonance lifetime. A clear illustration of this effect is the case mentioned above for $\nu^{\prime}=35$, that $\tau_{k=0}$ is enhanced from 3.8 ps to 61.0 ps when the resonance energy $E_{\mathrm{b}}=-57.75 \mathrm{~cm}^{-1}$ is excited, while it is only enhanced up to $37.0 \mathrm{ps}$ when the energy $E_{\mathrm{b}}=-60.76 \mathrm{~cm}^{-1}$ (with a lower spectrum intensity associated) is exccited. The magnitude of the $C_{E^{\prime}}^{\left(E_{\mathrm{b}}\right)}(t)$ coefficients also explains why the lifetime enhancement is maximized when $E_{\mathrm{b}}$ coincides with a resonance energy, and why a stronger overlap between resonances, which is expected to increase the spectrum intensity, favors the intensity and efficiency of lifetime control.

The energy-resolved $\mathrm{Br}_{2}\left(\mathrm{~B}, \nu_{\mathrm{f}}=\nu^{\prime}-2\right)$ fragment populations associated with the ground resonance energies $E_{\mathrm{a}}=61.8 \mathrm{~cm}^{-1}$ 
for $\nu^{\prime}=27$ and $E_{\mathrm{a}}=56.34 \mathrm{~cm}^{-1}$ for $\nu^{\prime}=35$, obtained when the three different $E_{\mathrm{b}}$ energies are excited, are displayed in Fig. 6(b) and $7(\mathrm{~b})$, respectively. For the sake of the clarity of the figures only the $\nu_{\mathrm{f}}=\nu^{\prime}-2$ population is shown, but the remaining $\mathrm{Br}_{2}\left(\mathrm{~B}, v_{\mathrm{f}}\right)$ populations display the same behavior with $E_{\mathrm{b}}$ as that of $\nu^{\prime}-2$. Similarly to the resonance survival probability, the behavior of the fragment distribution also depends on the choice of $E_{\mathrm{b}}$, and again both $\nu^{\prime}=27$ and 35 show the same trend. In this case the intensity of the interference effect in the fragment populations does not appear to depend only on the corresponding excitation spectrum intensity, but also on the proximity of $E_{\mathrm{b}}$ to the energy $E_{\mathrm{a}}$ for which the fragment distribution is calculated. Indeed, for $\nu^{\prime}=27$ the intensity of the interference effect is similar for $E_{\mathrm{b}}=-60.63$ and $-60.85 \mathrm{~cm}^{-1}$, while it increases appreciably for $E_{\mathrm{b}}=-61.12 \mathrm{~cm}^{-1}$, which is closer to the energy $E_{\mathrm{a}}=$ $-61.8 \mathrm{~cm}^{-1}$. The trend is more clear for $\nu^{\prime}=35$ where, with respect to the result obtained for $E_{\mathrm{b}}=-57.75 \mathrm{~cm}^{-1}$, the interference intensity decreases remarkably (by about a factor of two) when $E_{\mathrm{b}}$ gets far away from $E_{\mathrm{a}}$ to $E_{\mathrm{b}}=-60.76 \mathrm{~cm}^{-1}$, while in contrast the intensity increases by a factor of three for the closer energy $E_{\mathrm{b}}=-57.10 \mathrm{~cm}^{-1}$. The spectral intensity associated with the two additional $E_{\mathrm{b}}$ energies is lower, both for $\nu^{\prime}=27$ and 35, than that associated with the resonance energies $E_{\mathrm{b}}=-60.63$ and $-57.75 \mathrm{~cm}^{-1}$.

The explanation of the behavior of the intensity of the interference effect in the fragment distribution lies in eqn (2), (6), and (11). As reflected by eqn (2), a stationary eigenfunction $\psi_{E}(t)$ can be expressed as a superposition of continuum fragment states $\varphi_{E^{\prime}, m^{\prime}}$ with amplitudes $B_{E, m^{\prime}}^{(E)}$. It is expected that the maximum amplitude coefficients will be those associated with the energy $E^{\prime}=E, B_{E, m^{\prime}}^{(E)}$, and that the magnitude of $B_{E^{\prime}, m^{\prime}}^{(E)}$ will gradually decrease as $E^{\prime}$ gets far away from $E$. Thus, when a given energy $E_{\mathrm{b}}$ is excited (with a narrow bandwidth around it), in the corresponding eigenfunction $\psi_{E_{\mathrm{b}}}(t)$ the maximum amplitudes are $B_{E_{\mathrm{b}}, m^{\prime}}^{\left(E_{\mathrm{b}}\right)}$.

As discussed above, the long-delayed third pulse of $\mathcal{E}_{3}(t)$ that excites the $E_{\mathrm{b}}$ energy produces temporarily the amplitude $b_{E_{\mathrm{a}}, m^{\prime}}^{\left(E_{\mathrm{b}}\right)}(t)=\int \mathrm{d} E^{\prime \prime} C_{E^{\prime \prime}}^{\left(E_{\mathrm{b}}\right)}(t) B_{E_{\mathrm{a}}, m^{\prime}}^{\left(E^{\prime \prime}\right)}$ (see eqn (6), (10), and (11)) that interferes with the asymptotic $b_{E_{\mathrm{a}}, m^{\prime}}^{\left(E_{\mathrm{a}}\right)}(t)$ amplitude previously generated by the first pulse of $\mathcal{E}_{3}(t)$. The dependence again on the $C_{E^{\prime \prime}}^{\left(E_{\mathrm{b}}\right)}(t)$ coefficients indicates that the interference effect in the fragment distribution depends also on the spectral intensity at energy $E_{\mathrm{b}}$, as in the case of the survival probability. But $b_{E_{\mathrm{a}}, m^{\prime}}^{\left(E_{\mathrm{b}}\right)}(t)$ depends on the magnitude of the $B_{E_{\mathrm{a}}, m^{\prime}}^{\left(E^{\prime \prime}\right)}$ coefficients as well. And the $B_{E_{\mathrm{a}}, m^{\prime}}^{\left(E^{\prime \prime}\right)}$ coefficients in the superposition of the $\psi_{E_{\mathrm{b}}}(t)$ eigenfunction (and of those in the narrow bandwidth around) populated by the third pulse of $\mathcal{E}_{3}(t)$ will be higher - thus making also the $b_{E_{\mathrm{a}}, m^{\prime}}^{\left(E_{\mathrm{b}}\right)}(t)$ amplitudes in eqn (11) higher - as long as $E_{\mathrm{b}}$ becomes closer to $E_{\mathrm{a}}$. This explains the trend found in the results of Fig. 6(b) and 7(b) for the intensity of the interference effect. The dependence of $b_{E_{\mathrm{a}}, m^{\prime}}^{\left(E_{\mathrm{b}}\right)}(t)$ on the spectral intensity at $E_{\mathrm{b}}$ also explains the similarity of the interference intensity found at the two energies $E_{\mathrm{b}}=-60.63$ and $-60.85 \mathrm{~cm}^{-1}$ for $\nu^{\prime}=27$, because the smaller separation between $E_{\mathrm{a}}$ and $E_{\mathrm{b}}=-60.85 \mathrm{~cm}^{-1}$ is probably compensated by the lower spectral intensity at that energy compared to $E_{\mathrm{b}}=-60.63 \mathrm{~cm}^{-1}$.

The results of Fig. 6 and 7 have an interesting implication. From the theory developed above it is clear that control can be selectively exerted on each of the two resonance properties by properly choosing the magnitude of the delay time between the pulses that excite the two interfering energies $E_{\mathrm{a}}$ and $E_{\mathrm{b}}$. This temporal selectivity is related to the nature and time scale of the properties: the resonance survival probability and the associated lifetime are relatively short-time scale properties, while the fragment distribution is an asymptotic, long-time scale property. But in addition to this generic dependence of the control of the delay time between pulses, there is a strong dependence of the degree and efficiency of the control of the location of the second energy excited to induce the interference. And this dependence is different for the two resonance properties. The clear implication is that it is possible to optimize the control of the two properties by choosing properly a different $E_{\mathrm{b}}$ energy for each of them: optimization of lifetime control requires to maximize the spectral intensity at $E_{\mathrm{b}}$, regardless of the separation between $E_{\mathrm{a}}$ and $E_{\mathrm{b}}$, while optimal fragment distribution control requires minimization of the separation between $E_{\mathrm{a}}$ and $E_{\mathrm{b}}$, but also keeping high enough spectral intensity at $E_{\mathrm{b}}$.

\section{Conclusions}

A unified, single weak-field control scheme is suggested to modify the whole behavior of a resonance state, which is determined by the resonance lifetime and the fragment distribution produced upon resonance decay. The control scheme is based on quantum interference between overlapping resonances induced by the excitation of two (or more) different energies at which several resonances overlap. More specifically, in its simplest version the scheme applies a laser field consisting of three pulses, where the first pulse excites the energy of the resonance to be controlled; the second pulse excites another energy where the resonance under control overlaps with other resonance (or resonances) at a relatively short delay time with respect to the first pulse, inducing interference between the overlapping resonances which leads to resonance lifetime control; and the third pulse excites the same (or a different) energy as the second pulse, but now at a very long delay time with respect to the first pulse, inducing interference between the asymptotic fragment continuum states populated at both energies which leads to the fragment distribution control. Thus, with a single laser field the two resonance properties can be controlled just by varying the delay time of the pulses applied to modify each property.

The scheme is applied to two different situations where the intensity of resonance overlap is relatively weak and stronger. In both cases the control scheme works in a qualitatively similar way, although the interference effect becomes more intense as the overlap intensity increases, making the scheme more efficient. The degree and efficiency of the control achieved are shown to 
depend strongly on the choice of the second energy excited to induce interference with the energy of the resonance to be controlled. This dependence is found to be different for the two resonance properties, which implies that the control over each property can be optimized by choosing properly a different interfering energy in each case.

The formal theory underlying the unified interference control scheme is developed and presented. Such a theory explains all the results found when the control scheme is applied in different situations, and allows the design of the suitable conditions for the optimal control of the resonance properties. Due to the simplicity of the scheme, a wide application to control resonance-mediated molecular processes is envisioned.

\section{Conflicts of interest}

There are no conflicts of interest to declare.

\section{Acknowledgements}

This work was funded by the Ministerio de Economía y Competitividad (MINECO, Spain), Grant No. CTQ2015-65033-P, and the COST Action program, Grant No. CM1401 and CM1405. The Centro de Supercomputación de Galicia (CESGA, Spain) is acknowledged for the use of its resources.

\section{References}

1 D. W. Chandler, J. Chem. Phys., 2010, 132, 110901.

2 N. Balakrishnan, J. Chem. Phys., 2016, 145, 150901.

3 G. Balerdi, J. Woodhouse, A. Zanchet, R. de Nalda, M. L. Senent, A. García-Vela and L. Bañares, Phys. Chem. Chem. Phys., 2016, 18, 110.

4 A. García-Vela and K. C. Janda, J. Chem. Phys., 2006, 124, 034305. 5 A. García-Vela, J. Chem. Phys., 2008, 129, 094307.

6 C. M. Lovejoy and D. J. Nesbitt, J. Chem. Phys., 1990, 93, 5387.

7 R. T. Skodje, D. Skouteris, D. E. Manolopoulos, S. H. Lee, F. Dong and K. Liu, Phys. Rev. Lett., 2000, 85, 1206.

8 M. Qiu, Z. Ren, L. Che, D. Dai, S. A. Harich, X. Wang and X. Yang, Science, 2006, 311, 1440.

9 L. Che, et al., Science, 2007, 317, 1061.

10 J. B. Kim, et al., Science, 2015, 349, 510.

11 W. Shiu, J. J. Lin and K. Liu, Phys. Rev. Lett., 2004, 92, 103201. 12 T. Westermann, et al., Angew. Chem., Int. Ed., 2014, 53, 1122.

13 S. Bhattacharyya, S. Mondal and K. Liu, J. Phys. Chem. Lett., 2018, 9, 5502.

14 S. Chefdeville, et al., Phys. Rev. Lett., 2012, 109, 023201.

15 A. B. Henson, S. Gersten, Y. Shagam, J. Narevicius and E. Narevicius, Science, 2012, 338, 234.

16 E. Lavert-Ofir, et al., Nat. Chem., 2014, 6, 332.

17 S. N. Vogels, et al., Phys. Rev. Lett., 2014, 113, 263202.
18 S. N. Vogels, et al., Science, 2015, 350, 787.

19 J. Jankunas, K. Jachymski, M. Hapka and A. Osterwalder, J. Chem. Phys., 2015, 142, 164305.

20 A. Bergeat, J. Onvlee, C. Naulin, A. van der Avoird and M. Costes, Nat. Chem., 2015, 7, 349.

21 C. Naulin and M. Costes, Chem. Sci., 2016, 7, 2462.

22 D. Tannor and S. Rice, J. Chem. Phys., 1985, 83, 5013.

23 P. Brumer and M. Shapiro, Chem. Phys. Lett., 1986, 126, 541.

24 A. Assion, T. Baumert, M. Bergt, T. Brixner, B. Kiefer,

V. Seyfried, M. Strehle and G. Gerber, Science, 1998, 282, 919.

25 P. Anfinrud, R. de Vivie-Riedle and V. Engel, Proc. Natl. Acad.

Sci. U. S. A., 1999, 96, 8328.

26 R. J. Levis, G. M. Menkir and H. Rabitz, Science, 2001, 292, 709.

27 E. Skovsen, M. Machholm, T. Ejdrup, J. Thøgersen and

H. Stapelfeldt, Phys. Rev. Lett., 2002, 89, 133004.

28 C. Daniel, J. Full, L. González, C. Lupulescu, J. Manz, A. Merli, S. Vajda and L. Wöste, Science, 2003, 299, 536.

29 B. J. Sussman, D. Townsend, M. I. Ivanov and A. Stolow, Science, 2006, 314, 278.

30 G. Katz, M. A. Ratner and R. Kosloff, Phys. Rev. Lett., 2007, 98, 203006.

31 M. Shapiro and P. Brumer, Quantum Control of Molecular Processes, 2nd edn, Wiley-VCH verlag GmbH and Co. KGaA, Singapore, 2012.

32 F. Calegari, et al., Science, 2014, 346, 336.

33 R. Cireasa, et al., Nat. Phys., 2015, 11, 654.

34 M. E. Corrales, R. de Nalda and L. Bañares, Nat. Commun., 2017, 8, 1345.

35 A. García-Vela, J. Chem. Phys., 2012, 136, 134304.

36 A. García-Vela, J. Phys. Chem. Lett., 2012, 3, 1941.

37 A. García-Vela, J. Chem. Phys., 2013, 139, 134306.

38 A. García-Vela, Phys. Chem. Chem. Phys., 2015, 17, 29072.

39 A. García-Vela, Phys. Chem. Chem. Phys., 2018, 20, 3882.

40 A. García-Vela and N. E. Henriksen, J. Phys. Chem. Lett., $2015,6,824$.

41 A. García-Vela and N. E. Henriksen, Phys. Chem. Chem. Phys., 2016, 18, 4772.

42 A. García-Vela, Phys. Chem. Chem. Phys., 2016, 18, 10346.

43 A. García-Vela, J. Chem. Phys., 2016, 144, 141102.

44 A. García-Vela, Phys. Rev. Lett., 2018, 121, 153204.

45 J. C. Juanes-Marcos and A. García-Vela, J. Chem. Phys., 2000, 112, 4983.

46 J. A. Cabrera, C. R. Bieler, B. C. Olbricht, W. E. van der Veer and K. C. Janda, J. Chem. Phys., 2005, 123, 054311.

47 M. A. Taylor, J. M. Pio, W. E. van der Veer and K. C. Janda, J. Chem. Phys., 2010, 132, 104309.

48 C. Cohen-Tannoudji, B. Diu and F. Laloë, Quantum Mechanics, John Wiley and Sons, ch. XIII, vol. II, 1977.

49 A. García-Vela, Chem. Sci., 2017, 8, 4804.

50 P. Brumer and M. Shapiro, Chem. Phys., 1989, 139, 221.

51 A. García-Vela, J. Chem. Phys., 2007, 126, 124306. 